\title{
Theoretical study on the influence of twist Angle on the rate of reverse intersystem crossing (RISC) in D-A molecule
}

\author{
Mei Zhao ${ }^{1}$, Yao Guo ${ }^{1}$, Chang Li $^{1}$, Bing Yang ${ }^{2}$, and Yuyu Pan ${ }^{1}$ \\ ${ }^{1}$ Shenyang University of Technology \\ ${ }^{2}$ Jilin University
}

July 28, 2020

\begin{abstract}
Abstract:Efficient reverse intersystem crossing (RISC) is one of the most effective ways to achieve high exciton utilization of pure organic electroluminescent materials. There are two factors affecting the RISC rate (kRISC): the energy difference between the singlet and triplrt excited states $(\triangle \mathrm{ES}-\mathrm{T})$ and the spin-orbit coupling (SOC) between the excited states. In this article, based on the theoretical calculation method which can accurately and quantitatively describe the excited state of the molecule, the typical D-A molecule TPA-NZP is used as a template to study the change of the $\triangle \mathrm{ES}-\mathrm{T}$ and the SOC by adjusting the twisting angle of the donor and acceptor in the molecule. By studying the relationship between the excited state transition properties and the SOC, we find that different transition states have a great influence on the coupling. The two excited states are both LE states, but if the phase of the electron cloud are different, which will cause the SOC between them increased greatly; when the transition is the CT state, the SOC matrix elements between the LE state and the CT state both very small; when the singlet and triplet transitions occur in the same part, but the direction of the transition is changed may lead to a huge increase in the SOC.
\end{abstract}

Theoretical study on the influence of twist Angle on the rate of reverse intersystem crossing (RISC) in D-A molecule

Mei Zhao, ${ }^{\mathrm{a}}$ Yao Guo, ${ }^{\mathrm{a}}$ Chang Li, ${ }^{\mathrm{a}}$ Bing Yang ${ }^{\mathrm{b}}$ and Yuyu Pan ${ }^{\mathrm{a} *}$

Correspondence to: Yuyu Pan ( pyy39518768@163.com)

${ }^{a}$ School of Petrochemical Engineering, Shenyang University of Technology, 30 Guanghua Street, Liaoyang, 111003, P. R. China.

${ }^{b}$ State Key Laboratory of Supramolecular Structure and Materials, Jilin University, Changchun, 130012, P. R. China.

Abstract: Efficient reverse intersystem crossing (RISC) is one of the most effective ways to achieve high exciton utilization of pure organic electroluminescent materials. There are two factors affecting the RISC rate $(k$ RISC $)$ : the energy difference between the singlet and triplrt excited states $\left(\Delta \mathrm{E}_{\mathrm{S}-\mathrm{T}}\right)$ and the spin-orbit coupling (SOC) between the excited states. In this article, based on the theoretical calculation method which can accurately and quantitatively describe the excited state of the molecule, the typical D-A molecule TPANZP is used as a template to study the change of the $\Delta \mathrm{E}_{\mathrm{S}-\mathrm{T}}$ and the SOC by adjusting the twisting angle of the donor and acceptor in the molecule. By studying the relationship between the excited state transition properties and the SOC, we find that different transition states have a great influence on the coupling. The two excited states are both LE states, but if the phase of the electron cloud are different, which will cause the SOC between them increased greatly; when the transition is the CT state, the SOC matrix elements 
between the LE state and the CT state both very small; when the singlet and triplet transitions occur in the same part, but the direction of the transition is changed may lead to a huge increase in the SOC.

Key words: Hybrid local-charge transfer; Excited state; SOC; TD-DFT

\section{INTRODUCTION}

To achieve ultra-high exciton utilization, the reverse intersystem crossing (RISC) of excitons is the most effective method. ${ }^{[1-6]}$ In theory, the RISC process can be accounted for by the rate of the RISC $\left(k_{R I S C}\right)$ is estimated by following equations:

\section{Hosted file}

image1.wmf available at https://authorea.com/users/346859/articles/472687-theoretical-studyon-the-influence-of-twist-angle-on-the-rate-of-reverse-intersystem-crossing-risc-in-da-molecule

where is spin-orbit coupling constant (SOC) between the crucial excited singlet $(\mathrm{S})$ and triplet $(\mathrm{T})$ states in the organic molecules, $\Delta E_{\Sigma T}$ is energy difference between $\mathrm{S}$ and $\mathrm{T}$ states, $\mathrm{t}$ is the temperature, and $k_{b}$ refer to the Boltzmann constant, $\lambda$ is the hole/electron reorganization energy calculated by using the adiabatic potential energy surfaces method. According to the formula, the value of the $k_{R I S C}$ is related to the $\Delta E_{\Sigma T}$ and the SOC, both of which are proportional to the rate. And these two parameters are closely related to the transition properties in the excited-state.

The TPA-NZP molecule is a typical "Hot Excition" mechanism, with an ultra-high exciton utilization efficiency of $\sim 93 \%$. Its excited state has unique features, such as a huge triplet state energy gap $\left(\Delta \mathrm{E}_{\mathrm{T} 1-\mathrm{T} 2}\right)$ between the first $\left(T_{1}\right)$ and the second $\left(T_{2}\right)$ triplet excited-state and nearly degenerate the second singlet and triplet states, $\left(\Delta \mathrm{E}_{\mathrm{S} 2-\mathrm{T} 2}-0\right)$. Its caused the IC $\left(\mathrm{T}_{2}-\mathrm{T}_{1}\right)$ rate so slow that no enough to compete with the $k_{R I S C}$ from the $\mathrm{T}_{2}$ to $\mathrm{S}_{2}$, thus ensuring the smooth of the high exciton channel. In addition, the $\mathrm{S}_{1}$ state of the TPA-NZP molecule is called a Hybrid Local-Charge Transfer (HLCT) excited state. The most notable feature of this system is that the HLCT state is formed by the hybridization of the intrinsic LE state and CT state of the molecule, and has both the LE state and the CT state feature. ${ }^{[7-8]}$

Observing the transition process of excited states by a large number of calculation results, we found that the excited state of the most molecules is not a complete localized excited (LE) state or a complete chargetransfer (CT) state. Most contain both CT-state components and LE-state components. ${ }^{[9-11]}$ Such molecules exhibit different excited state properties due to their different ratios of LE and CT states (for example, local states dominated by LE states or charge transfer states dominated by CT states). When the ratio of the LE state or the CT state reaches a certain level, the excited state will exhibit a new properties, it is different from the classical excited state, and which is also different from the mixed state of the traditional double fluorescent molecule.

In previous work, we have discussed in detail the excited state energies of the TPA-NZP molecule, ${ }^{[7-9]}$ however, the influence of this hybrid on the $\Delta E_{\Sigma T}$ and the SOC between the singlet and triplet states are rarely systematically analyzed. In the present study, we focus on the $\Delta E_{\Sigma T}$ and the SOC between the neighboring singlet and triplet excited states of the TPA-NZP, as the change of excited state transition properties. With the better understanding on the relationship between the excited states, it is very helpful for a rational molecular design to adjust and control the excited states, aiming at improve the excition utilization in the new-generation fluorescent OLED materials. We expect that our theoretical studied results can provide experimental researchers with a lot of useful information on designing and synthesizing new efficient OLED materials.

\section{COMPUTATION DETAILS}

Nowadays, the equation of motion coupled cluster with single and double excitations model (EOM-CCSD) 
methods and time-dependent density functional theory (TD-DFT) are very popular computational methods for the excited-state electronic structures. The EOM-CCSD method taking into account the both single and double substitutions, and it can give relatively accurate results, but the calculation cost is too high. ${ }^{[12]}$ In contrast, TD-DFT is a higher cost-effective method for calculated the excited-state, and it is widely used in the calculation of the excited state of medium-sized molecules. ${ }^{[13-16]}$ In the previous work, we have screened and verified the functional calculation of the excited state of the HLCT state molecule. The performance of 2 local functionals (SVWN ${ }^{[17]}$ and PBE ${ }^{[18]}$ ), 7 hybrid functionals (BLYP, ${ }^{[19]} \mathrm{B} 3 \mathrm{LYP},{ }^{[20]}$ PBE0, $\left.{ }^{[21]} \mathrm{BMK},{ }^{[22]} \mathrm{BHandHLYP},{ }^{[23]} \mathrm{M} 06-2 \mathrm{X},{ }^{[24]} \mathrm{M} 06 \mathrm{HF}^{[25]}\right)$, and 2 long-range-corrected functional CAMB3LYP ${ }^{[26]}$ and $\omega \mathrm{B} 97 \mathrm{X}^{[27]}$ in combination with $6-31+\mathrm{G}(\mathrm{d}, \mathrm{p})^{[28]}$ basis sets is explored. Among these methods, the RSH functionals TD-DFT/ $\omega \mathrm{B} 97 \mathrm{X}$ provided the results which were the closest to those data from EOM-CCSD and the experimental values. Therefore,we employ the TD-DFT/ $\omega$ B97X/ 6-31+G(d,p) method descript the excited-state. All DFT and EOM-CCSD calculations have been performed with the Gaussian09.D.01 revision. ${ }^{[29]}$ We also calculate the effective spin-orbit coupling (SOC) matrix elements between the singlet and triplet excited states by using two-component relativistic TD-DFT method implemented in the Beijing Density Functional (BDF) program. ${ }^{[30-31]}$ TD-DFT methods have been employed to calculate SOC matrix elements in many studies, and the results are in line with those given by DFT-MRCI method. Due to the functional limitation of this method, in this section we use the other long-range-corrected functional CAM-B3LYP functional to calculate the SOC.

\section{RESULTS AND DISCUSSION}

The molecular structure of TPA-NZP (Figure 1) is composed of the triphenylamine (TPA) which acts as a donor and the naphtho [2,3-c] $[1,2,5]$ thiadiazol (NZ) group which serves as an acceptor. A very small singlet-triplet energy split $\left(\Delta \mathrm{E}_{\mathrm{ST}}=0.29 \mathrm{eV}\right)$ between $\mathrm{S}_{2}$ and $\mathrm{T}_{2}$ due to the dominant CT character could cause a RISC through the "hot exciton" $\mathrm{S}_{2}$ and $\mathrm{T}_{2}$ states, instead of the "cold exciton" $\mathrm{S}_{1}$ and $\mathrm{T}_{1}$ states.

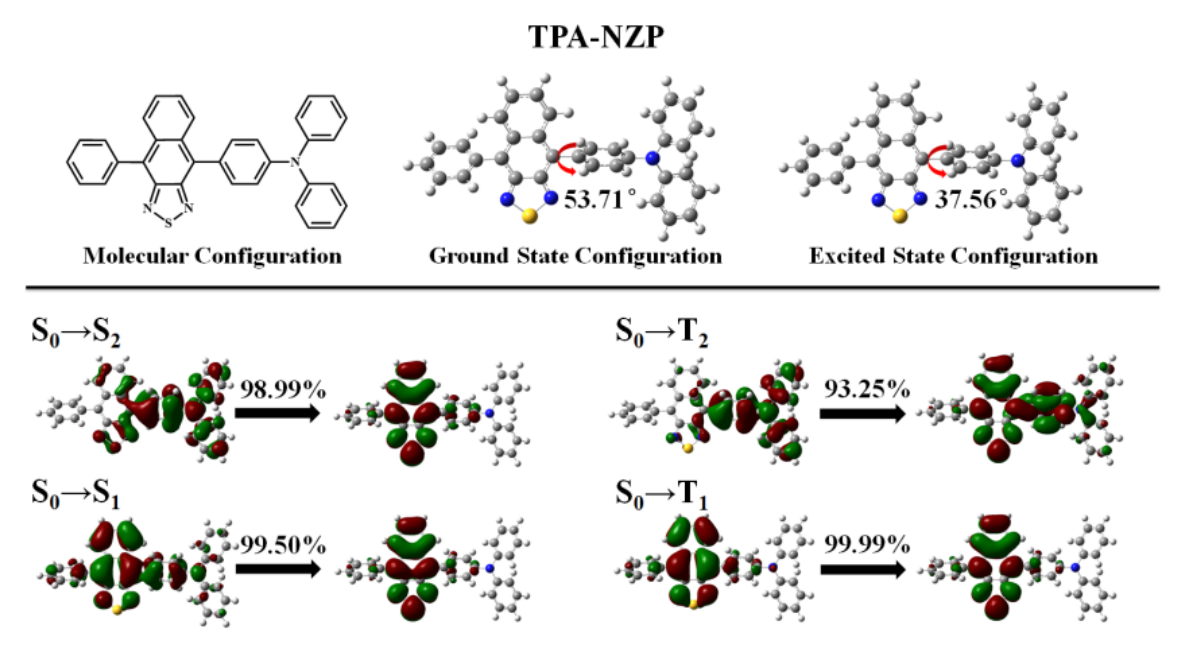

Figure 1. The molecular structure, ground and excited-state molecular configuration of the TPA-NZP; and the excited state transition properties of the $\mathrm{S}_{1}$ and $\mathrm{S}_{2}$ states.

\subsection{TRANSITION CHARACTER}

As shown in the Figure1, the first singlet excited-state is a HLCT state, but it can be seen from the figure that its transition is dominated by the LE state. After calculation, the transition dipole moment of the $\mathrm{S}_{1}$ state $\left(\mu \mathrm{s}_{1}\right)$ is 6.25 Debye (dipole moment of the $\mathrm{S}_{0}$ state $\left(\mu \mathrm{s}_{0}\right)$ is 1.72 Debye), which also confirms this inference. At the same time, the $\mathrm{S}_{2}$ state is also a HLCT state, but its transition is dominated by the CT state, and the transition dipole moment of the $\mathrm{S}_{2}$ state is 18.40 Debye. It can be seen that the proportion of $\mathrm{CT}$ in this transition is very large. 
In order to further explore the hybridization process of the excited state and its influencing factors, we fixed the twisting angle between the donor (TPA) and acceptor (NZ) moieties of the TPA-NZP molecule, and observed the changes of the excited states energies and the excited state transition properties at different dihedral angle.

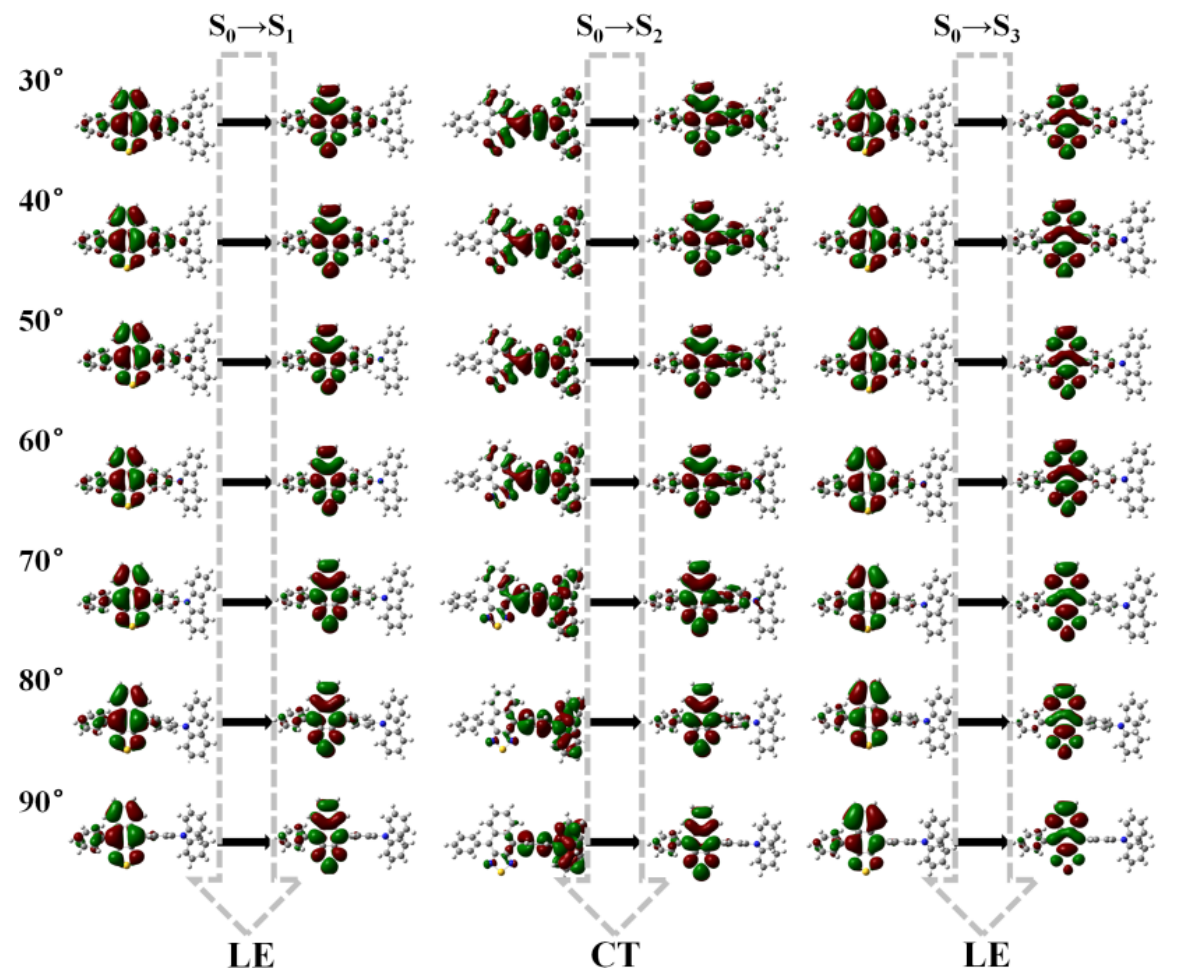

Figure 2. Natural transition orbit (NTOs) 32 analysis of the TPA-NZP molecular in the $\mathrm{S}_{1}, \mathrm{~S}_{2}$ and $\mathrm{S}_{3}$ excited states at different dihedral angle. 


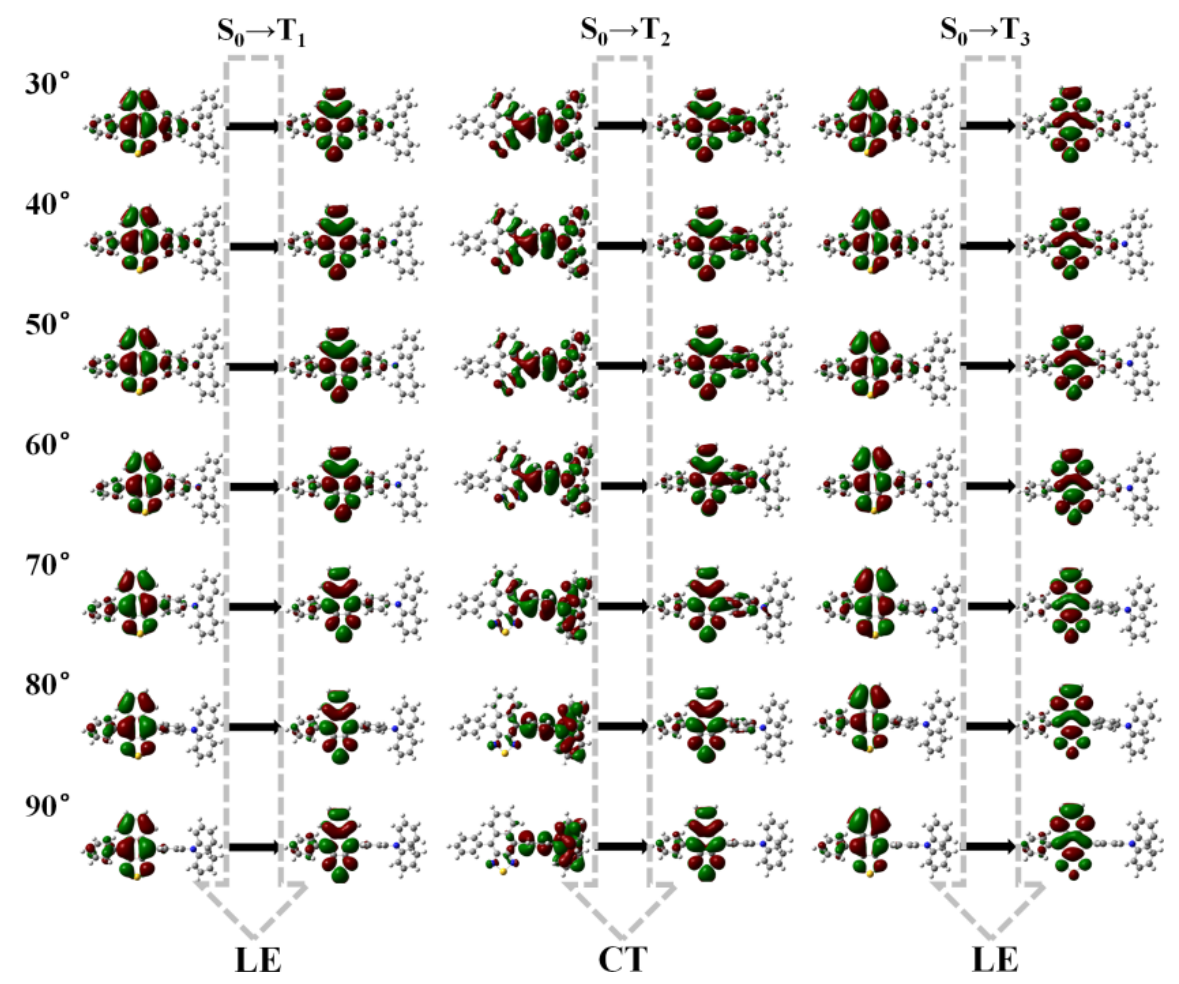

Figure 3. Natural transition orbit (NTOs) analysis of the TPA-NZP molecular in the $\mathrm{T}_{1}, \mathrm{~T}_{2}$ and $\mathrm{T}_{3}$ excited states at different dihedral angle.

As shown in Figure 2, as the twisting angle was increased from $30^{\circ}$ to $90^{\circ}$, the $\mathrm{S}_{1}$ state changes from the HLCT state which dominated by the LE state to the complete LE state, and the $\mathrm{S}_{2}$ state changes from the HLCT state which dominated by the CT state to the almost complete CT state, the change in the $\mathrm{S}_{3}$ states are smaller than the first two excited states. The transition property of the first three triplet excited states is similar to the single excited states with the increase of the twisting angle, shown in Figure 3. 


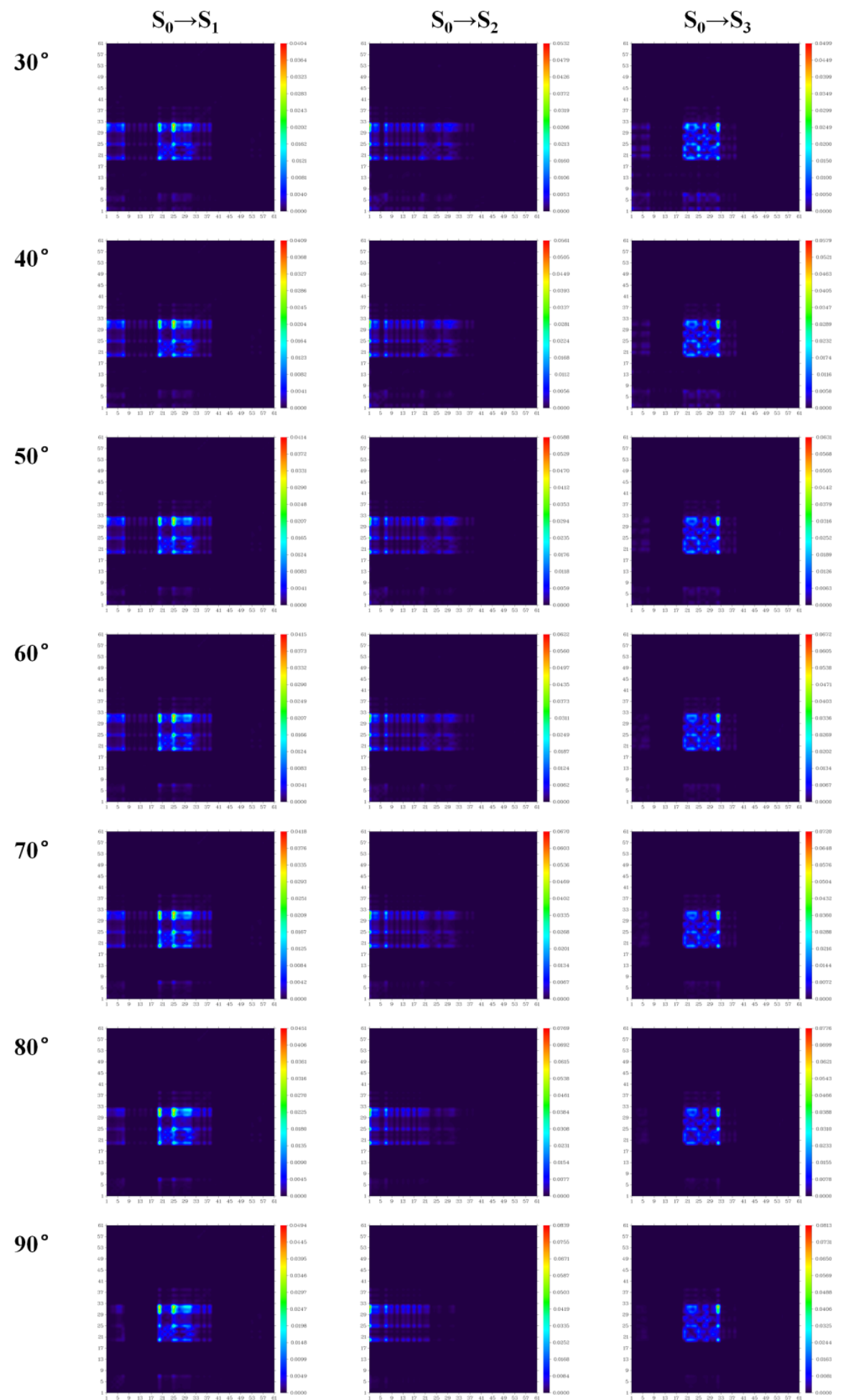

Figure 4. The transition density matrix map of the $S_{1}, S_{2}$ and $S_{3}$ for the molecules with difference twisting angle.

We also calculate the wave functions of electron-hole pairs from the transition density matrix, and plot them 
in a two-dimension (2D) color-filled maps, which is related to the probability of finding the electron and hole in the atomic orbitals localized on each non-hydrogen atom, used the Multiwfn software 3.6 revision, ${ }^{[33]}$ shown in Figure 4. From this map we can understand which atoms are mainly affected by the electron transition and which atom pairs are strongly coherent when electron transits. The effect is stronger during the transition the value is brighter in the map. The diagonal part represents the LE component localized on the main backbone, while the off-diagonal region denotes the CT component. 'The maps of the Figure 4 show that the values of the $\mathrm{S}_{1}, \mathrm{~S}_{2}$ and the $\mathrm{S}_{3}$ transition have much difference, for example, the $\mathrm{S}_{1}$ state gradually darkens as the twisting angle increases, indicating that the composition of the CT state gradually decreases during the transition, while the $\mathrm{S}_{2}$ state gradually dims the brightness in the diagonal direction as the twisting angle increases, at the $90^{\circ}$, the map is almost concentrated in the opposite diagonal direction, which means that the transitions are almost all the CT components, whereas the transitions of the $\mathrm{S}_{3}$ state are all on the NZ molecule, most of which are LE transitions. The characteristics reflected from the transition density matrix are almost as same as the NTOs in the Figure 2.
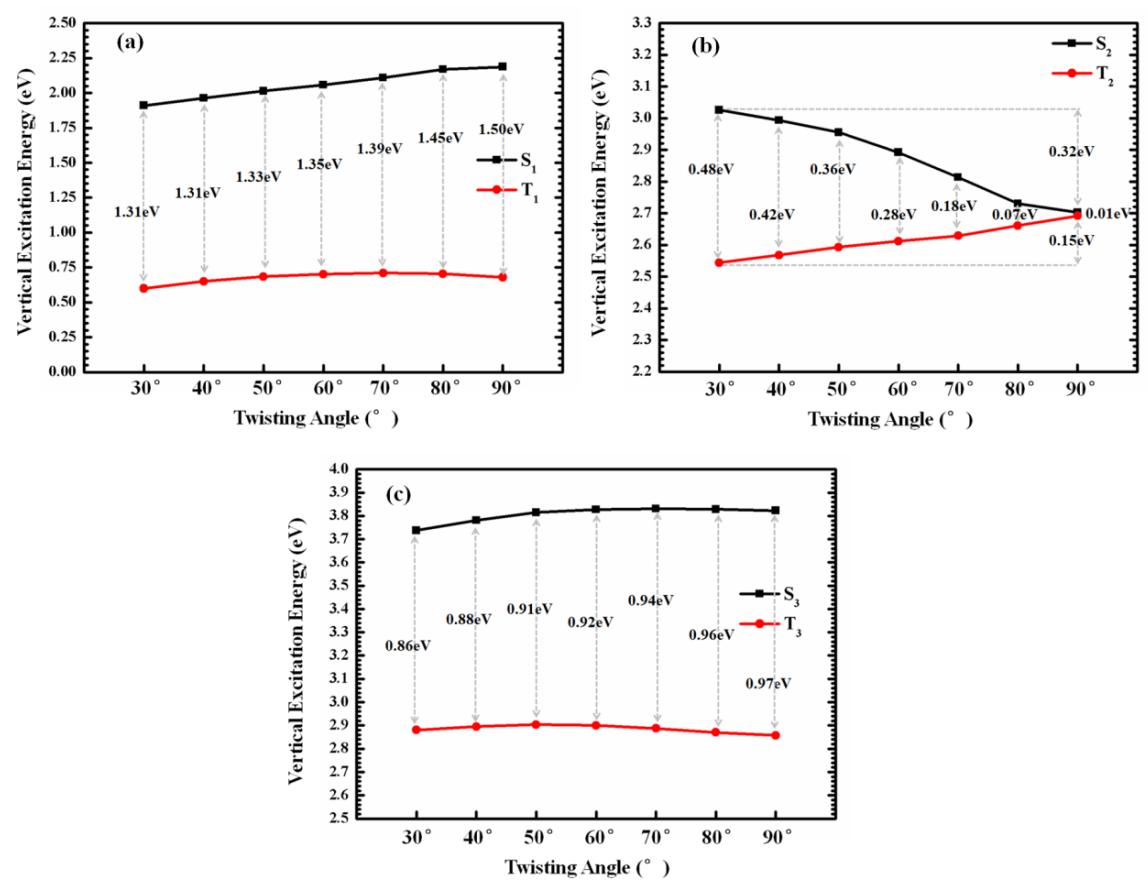

Figure 5. The change of excited state energy at different dihedral angles in the $\mathrm{S}_{1}, \mathrm{~S}_{2}$ and $\mathrm{S}_{3}$ excited states and the $\mathrm{T}_{1}, \mathrm{~T}_{2}$ and $\mathrm{T}_{3}$ excited states.

In terms of excitation energy, as shown in Figure 5, in the $S_{1}$ state, the transition ratio of the LE state increases with the increase of the twisting angle from $30^{\circ}$ to $90^{\circ}$, and the energy also increases about $0.2 \mathrm{eV}$, but the energy change of the $T_{1}$ state is relatively small, which is about $0.08 \mathrm{eV}$, which leads to the energy difference between them $\left(\Delta \mathrm{E}_{\mathrm{S} 1-\mathrm{T} 1}\right)$ increases from $1.31 \mathrm{eV}$ to $1.50 \mathrm{eV}$. On the contrary, with the twisting angle increases, the energy of the $\mathrm{S}_{2}$ state $\left(\mathrm{E}_{\mathrm{S} 2}\right)$ and $\mathrm{T} 2$ state $\left(\mathrm{E}_{\mathrm{T} 2}\right)$ are change greatly. The transition ratio of the CT state become larger, the energy change is also relatively large. From $30^{\circ}$ to $90^{\circ}$, the $\mathrm{E}_{\mathrm{S} 2}$ is reduced about $0.32 \mathrm{eV}$, the $\mathrm{E}_{\mathrm{T} 2}$ increased about $0.15 \mathrm{eV}$, the $\Delta \mathrm{E}_{\mathrm{S} 2 \text { - } \mathrm{T} 2}$ reduced from $0.48 \mathrm{eV}$ to $0.01 \mathrm{eV}$, at the same time, the $\mathrm{E}_{\mathrm{S} 3}$ and $\mathrm{E}_{\mathrm{T} 3}$ have very small changes due to the small change of transition character, are about $0.08 \mathrm{eV}$ and $0.02 \mathrm{eV}$ respectively. The changes indicate that the transition participation of the CT state in the excited state has a great influence on the excitation energy, especially in the singlet state. The larger the proportion of the CT state, the lower the transition energy, but the ET is less affected by the transition property of the excited state and, the change trend of energy is opposite to the singlet state, the larger the 
ratio of $\mathrm{CT}$ state, the higher the excitation energy.

\subsection{SPIN-ORBIT COUPLING CONSTANT (SOC)}

On the basis of different twisting angles, we used BDF to calculate the spin-orbit coupling (SOC) matrix elements between the singlet and triplet excited states. Due to the functional limitations in the BDF program, we used CAM-B3LYP functional for subsequent calculation. However, as we all know, the functional dependence of the TD-DFT method is very strong, especially for the CT state, so the electronic cloud distribution of the excited state is different from the previous part, but has the same general trend, so we analyze the SOC by the TD-DFT/ CAM-B3LYP /6-31+G(d,p).

Firstly, we studied the SOC matrix elements between the singlet and triplet excited states all exhibit the LE character. As shown in Figure 6, the transitions process in the $\mathrm{S}_{0}-\mathrm{T}_{1}, \mathrm{~S}_{0}-\mathrm{S}_{1}$ and $\mathrm{S}_{0}-\mathrm{S}_{3}$ all occur in the NZ part, and there are little change with increasing the twist angle, which are almost complete LE state. For instance, the transitions of the $\mathrm{S}_{0}-\mathrm{S}_{1}$ are dominated by the LE state, but when the twist angle is small, the electron cloud has a small amount of distribution on the adjacent benzene ring of NZ, but as the twist angle increases, it gradually becomes the complete LE state, the SOC between the $T_{1}$ and $S_{1}$ states are relatively small (from about $0.14 \mathrm{~cm}^{-1}$ to $0.27 \mathrm{~cm}^{-1}$ ), and the maximum value is when the twist angle is $60^{\circ}$. When the transition is exactly the same, for example at $90^{\circ}$, the SOC is the smallest. However, the phase of the electron cloud distribution is different, the SOC increases greatly. For example, as shown in right side of the Figure 6 , the transition of the $\mathrm{S}_{0}-\mathrm{S}_{3}$ occur on the NZ too, but the phases of the "Hole" part are different, and the SOC constant between them can be seen that it has increased by almost 10 times compared with the fully same transition in the left of the same figure.

Secondly, contrary to the transition process of the $\mathrm{S}_{0}-\mathrm{S}_{1}$ state, the transition process of the $\mathrm{S}_{0}-\mathrm{S}_{2}$ state is the HLCT state dominated by the CT state. With the increase of the twist angle, it becomes an almost complete CT state at the 90deg. So, next we will study the SOC matrix elements between the CT state and LE state, the CT state and CT state. On the one hand, as shown in the left of Figure 7, the transitions process in the $\mathrm{S}_{0}-\mathrm{T}_{1}$ is almost a complete LE state, the SOC between the $\mathrm{S} 2$ and $\mathrm{T} 1$ states are very small, the maximum value is at the $60 \mathrm{deg}$, and the minimum value appears at the $80 \mathrm{deg}$, and these values are similar to the SOC between the LE state and the LE state transition where the transition is exactly the same. This shows that the SOC between the complete LE state, and between complete LE and CT is relatively small, but when the hybridization is moderate, it is helpful to increase the SOC value. On the other hand, as shown on the right side of Figure 7, with the twist angle increases, the transition of the $\mathrm{S}_{3}$ gradually changes from the HLCT state to the LE state located on triphenylamine. As the increases of the LE excited state proportion, the SOC also increases. At 90deg, the SOC between the $\mathrm{S}_{2}$ state of the complete CT state and the $\mathrm{S}_{4}$ state of the complete LE state has reached 1.0433. 


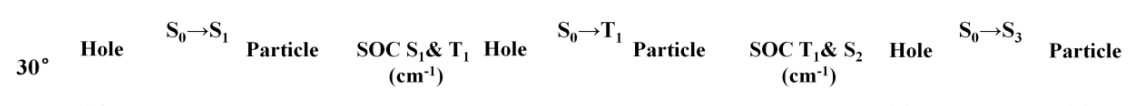

$$
\begin{aligned}
& { }_{40}^{\circ}, 0_{0}^{\circ} \text { - } \\
& { }_{50}^{\circ}
\end{aligned}
$$

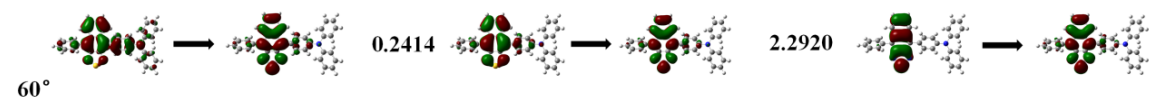

$$
\begin{aligned}
& { }_{70}^{\circ} \text { की } \\
& { }_{80}^{\circ}{ }^{\circ}
\end{aligned}
$$

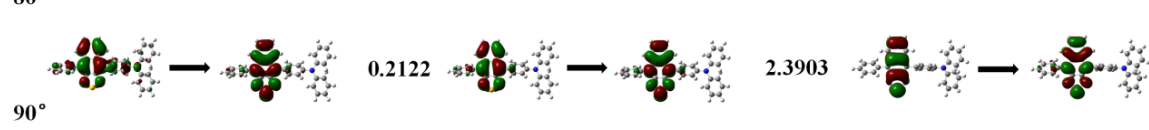

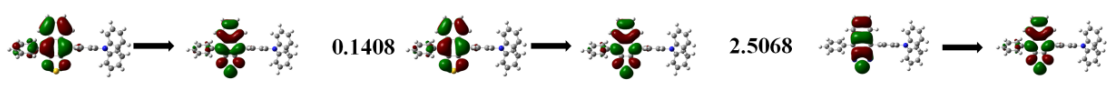

Figure 6. NTOs and SOC matrix elements $\left(\mathrm{cm}^{-1}\right)$ of the TPA-NZP molecular in different twisting angles of the $\mathrm{S}_{0}-\mathrm{S}_{1}, \mathrm{~S}_{0}-\mathrm{T}_{1}$ and $\mathrm{S}_{0}-\mathrm{S}_{2}$.

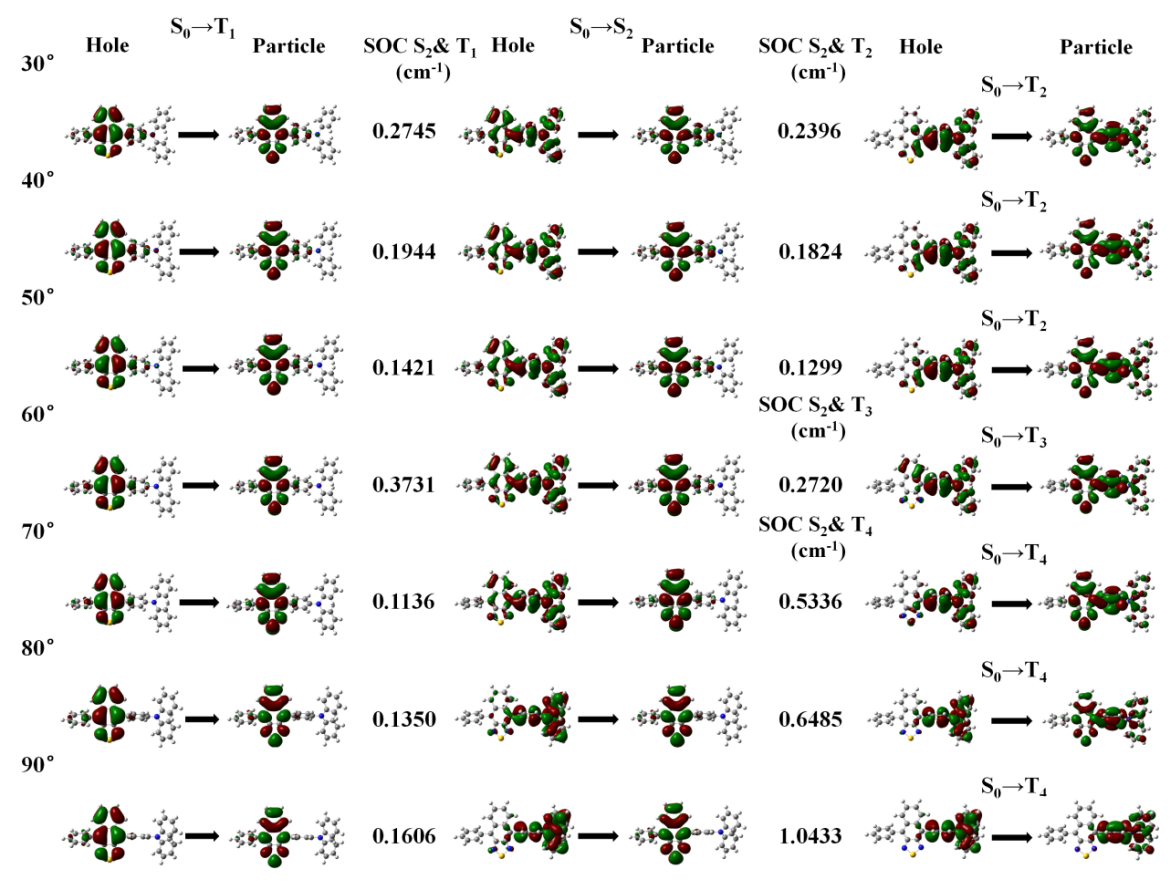

Figure 7. NTOs and SOC matrix elements $\left(\mathrm{cm}^{-1}\right)$ of the TPA-NZP molecular in different twisting angles of the $\mathrm{S}_{0}-\mathrm{T}_{1}, \mathrm{~S}_{0^{-}} \mathrm{S}_{2}$ and $\mathrm{S}_{0}-\mathrm{T}_{2}, \mathrm{~S}_{0}-\mathrm{T}_{3}$ and $\mathrm{S}_{0}-\mathrm{T}_{4}$

Thirdly, and the most significant, as shown on the left side of Figure 8, is the transition characteristics and coupling strength between the $\mathrm{T}_{4}$ and $\mathrm{S}_{4}$. We were surprised to find that the SOC constant between them 
is very large, reaching about $22 \mathrm{~cm}^{-1}$, and as the transitions of these two excited states do not change much with the twist angle, the SOC constants are kept at a higher value. It can be seen from the transition characteristics that the "Partical" parts of $\mathrm{T}_{4}$ and $\mathrm{S}_{4}$ are exactly the same, which the electronic clouds are localized in the NZ part, but the "Hole" part are very different. The $\mathrm{T}_{4}$ state transition occurs along the longitudinal direction, but the $\mathrm{S}_{4}$ state transition are from horizontal to vertical, which may be one of the reasons for the increase in SOC. However, the SOC constants between the $\mathrm{S}_{4}$ state and $\mathrm{S}_{5}$ state are very small, in which the electronic clouds in the $\mathrm{S}_{5}$ are localized in the triphenylamine part.

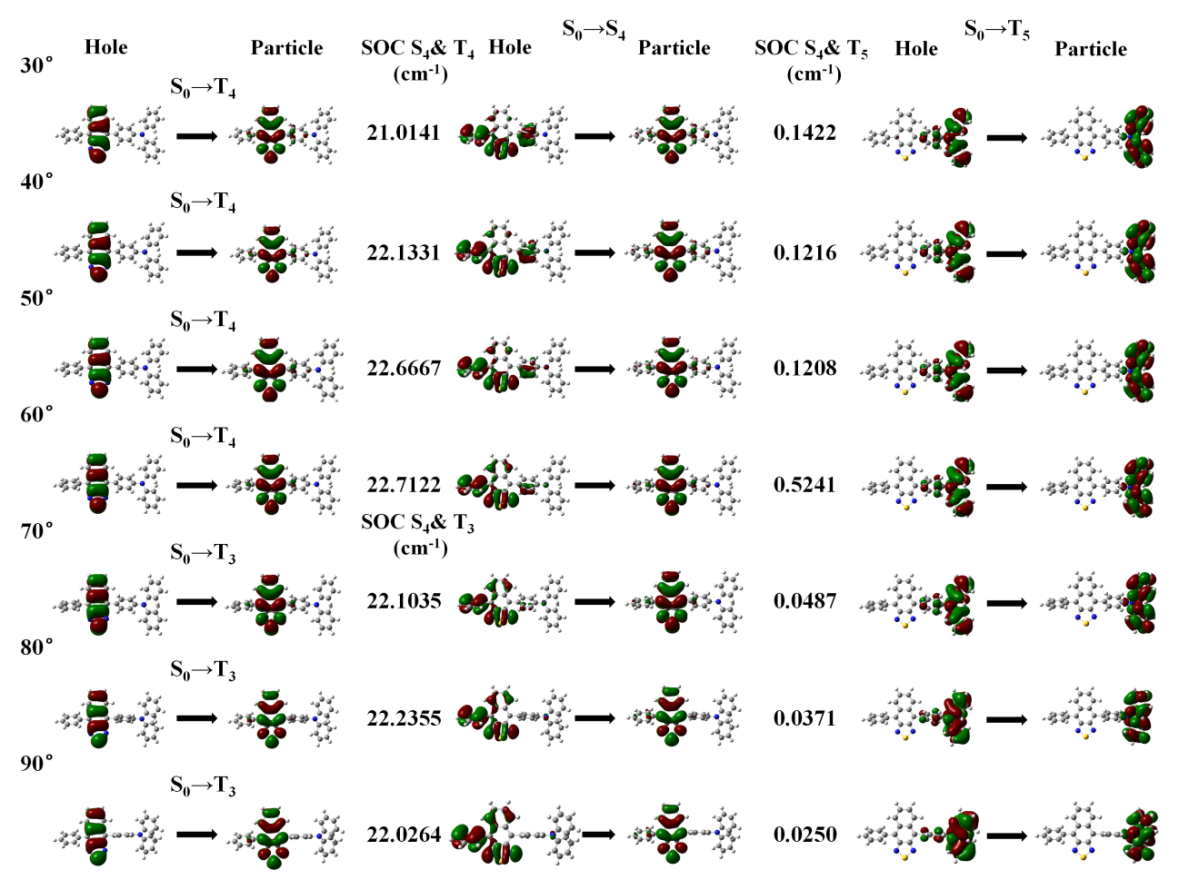

Figure 8. NTOs and SOC matrix elements $\left(\mathrm{cm}^{-1}\right)$ of the TPA-NZP molecular in different twisting angles of the $\mathrm{S}_{0}-\mathrm{T}_{3}, \mathrm{~S}_{0}-\mathrm{T}_{4}, \mathrm{~S}_{0}-\mathrm{S}_{4}$ and $\mathrm{S}_{0}-\mathrm{T}_{5}$.

\section{CONCLUSION}

In this article, we analyzed the two important parameters that affect the $k_{R I S C}$ by adjusting the twist angle in the molecule. Firstly, we found that the change of the twist angle has a great impact on the transitions that have partial CT states, but for the LE states has little effect. Secondly, we explored the changes of the SOC matrix elements under different excited state, and came to the following conclusions: 1). when the singlet state and the triplet state are both LE states and the electron cloud is exactly the same, the SOC matrix elements between them is very small, but when although the two excited states are both LE states, the phase of the electron cloud changes when the transition occurs, which will cause the SOC between them to increase greatly; 2). when the transition is dominated by the CT state, the SOC matrix elements between the LE state and the CT state both very small; 3). when the singlet and triplet transitions occur in the same part, but the direction of the transition is changed, for example, the transition from the horizontal axis to the vertical axis may lead to a huge increase in coupling. This finding will provide an idea for the design of adjustable energy molecules and provide a reference for the application of energy levels of triplet excited states in the future.

\section{FUNDING INFORMATION}

This work was supported by the Scientific Research Funding Project of Education Department of Liaoning Province (No. LQGD2019011) and the National Natural Science Foundation of China (No. 51603127). 


\section{REFERENCES}

[1] H. Uoyama, K. Goushi, K. Shizu, H. Nomura, C. Adachi,Nature, 2012 , 492 , 234.

[2] S. Y. Lee, T. Yasuda, Y. S. Yang, Q. S. Zhang, C. Adachi, Angew. Chem. Int. Ed. 2014,53 , 6402.

[3] J. Li, T. Nakagawa, J. MacDonald, Q. Zhang, H. Nomura, H. Miyazaki, C. Adachi, Adv. Mater. 2013 , 25,3319 .

[4] G. Méhes, H. Nomura, Q. Zhang, T. Nakagawa, C. Adachi, Angew. Chem. Int. Ed. 2012 ,51 , 11311.

[5] S. T. Zhang, W. J. Li, L. Yao, Y. Y. Pan, B. Yang, Y. G. Ma, F. Z. Shen, R. Xiao, Chem. Commun.2013 , 49,11302 .

[6] L. Yao, S. T. Zhang, R. Wang, W. J. Li, F. Z. Shen, B. Yang, Y. G. Ma, Angew. Chem. Int. Ed.2014, $126,2151$.

[7] W. J. Li, Y. Y. Pan, R. Xiao, Q. M. Peng, S. T. Zhang, D. G. Ma, F. Li, F. Z. Shen, Y. H. Wang, B. Yang, Y. G. Ma, Adv. Funct. Mater. 2014 , 24, 1609.

[8] Y. Y. Pan, W. J. Li, S. T. Zhang, L. Yao, C. Gu, H. Xu, B. Yang, Y. G. Ma, Adv. Opt. Mater.2014, 2 , 510 .

[9] H. C. Liu, Q. Bai, L. Yao, H. Y. Zhang, H. Xu, S. T. Zhang, W. J. Li, Y. Gao, J. Y. Li, P. Lu, H. Y. Wang, B.Yang, Chemical science Chem. Sci. 2015 , 6 , 3797.

[10] S. F. Xue, X. Qiu, S. A. Ying, Y. S. Lu, Y. Y. Pan, Q. K. Sun, C. Gu, W. J. Yang, Adv. Opt. Mater. $2017,5,1700747$.

[11] Y. W. Xu, X. M. Liang, X. H. Zhou, P. Yuan, J. D. Zhou, C. Wang, B. B. Li, D. H. Hu, X. F. Qiao, X. F. Jiang, L.L. Liu, S. J. Su, D. G. Ma, Y. G. Ma, Adv. Mater.2019, 31 , 1807388.

[12] P. Foggi, F. V. Neuwahl, R. L. Moroni, P. R. Salvi, J. Phys. Chem. A. 2003, 107, 1689.

[13] G. E. Scuseria, III, H. F. J. Schaefer J. Chem. Phys.1989, 90 , 3700.

[14] Y.Y. Pan, J. Huang, S. T. Zhang, D. W. Yu, B. Yang, Y. G. Ma, RSC Adv. 2016 , 6 , 108404.

[15] M. E. Casida, C. Jamorski, K. C. Casida, D. R. Salahub, J. Chem. Phys. 1998 ,108 , 4439.

[16] R. E. Stratmann, G. E. Scuseria, M. J. Frisch, J. Chem. Phys, 1998 ,109 , 8218.

[17] S. H. Vosko, L. Wilk, M. Nusair, Can. J. Phys.1980 ,58 , 1200.

[18] J. P. Perdew, K. Burke, M. Ernzerhof, Phys. Rev. Lett.1996 , 77 , 3865.

[19] B. Miehlich, A. Savin, H. Stoll, H. Preuss, Chem. Phys. Lett. 1989 , 157 , 200.

[20] A. D. Becke, J. Chem. Phys. 1993, $98,5648$.

[21] C. Adamo, V. Barone, J. Chem. Phys. $1999,110,6158$.

[22] A. D. Boese, J. M. L. Martin, J. Chem. Phys.2004, 121 , 3405.

[23] A. D. Becke, J. Chem. Phys. 1993 , 98 , 1372.

[24] Y. Zhao, D. G. Truhlar, Theor. Chem. Acc. 2008 ,120 , 215.

[25] Y. Zhao, D. G. Truhlar, J. Phys. Chem. A, 2006 ,110 , 13126.

[26] T. Yanai, D. Tew, N. Handy, Chem. Phys. Lett.2004, 393 , 51.

[27] J. D. Chai, M. H. Gordon, J. Chem. Phys, 2008 ,10 , 6615.

[28] V. A. Rassolov, J. A. Pople, M. A. Ratner, T. L. Windus, J. Chem. Phys. 1998 , 109 , 1223. 
[29] M. J. Frisch, G. W. Trucks, H. B. Schlegel, G. E. Scuseria, M. A. Robb, J. R. Cheeseman, G. Scalmani, V. Barone, B. Mennucci, G. A. Petersson, H. Nakatsuji, M. Caricato, X. Li, H. P. Hratchian, A. F. Izmaylov, J. Bloino, G. Zheng, J. L.Sonnenberg, M. Hada, M. Ehara, K. Toyota, R. Fukuda, J. Hasegawa, M. Ishida, T. Nakajima, Y. Honda, O. Kitao, H. Nakai, T. Vreven, J. A. Montgomery Jr., J. E.Peralta, F. Ogliaro, M. Bearpark, J. J. Heyd, E. Brothers, K. N. Kudin, V. N.Staroverov, R. Kobayashi, J. Normand, K. Raghavachari, A. Rendell, J. C. Burant, S. S. Iyengar, J. Tomasi, M. Cossi, N. Rega, J. M. Millam, M. Klene, J. E. Knox, J. B.Cross, V. Bakken, C. Adamo, J. Jaramillo, R. Gomperts, R. E. Stratmann, O.Yazyev, A. J. Austin, R. Cammi, C. Pomelli, J. W. Ochterski, R. L. Martin, K. Morokuma, V. G. Zakrzewski, G. A. Voth, P. Salvador, J. J. Dannenberg, S. Dapprich, A. D. Daniels, Ö. Farkas, J. B. Foresman, J. V. Ortiz, J. Cioslowski, D. J. Fox, Gaussian 09 (Revision D.01), Gaussian, Inc., Wallingford, CT, 2009.

[30] Z. Li, Y. Xiao, W. Liu, Mole. Phy. 2013 ,111, 3741.

[31] F. Wu, W. Liu, Y. Zhang, Z. Li, J. Chem. Theory Comput,2011 , 7, 3643.

[32] R. L. Martin, J. Chem. Phys. 2003 , $118,4775$.

[33] T. Lu, F. Chen, J. Comput. Chem.2012 ,33 , 580. 